\title{
Fock-space multi-reference coupled-cluster response with the effect of triples on dipole moment of $\mathrm{CIO}$ and SF radicals ${ }^{\#}$
}

\author{
LALITHA RAVICHANDRAN, DEBARATI BHATTACHARYA, NAYANA VAVAL \\ and SOURAV PAL* \\ Physical Chemistry Division, National Chemical Laboratory (CSIR), Pune 411 008, India \\ e-mail: s.pal@ncl.res.in
}

\begin{abstract}
Dipole moment calculations of $\mathrm{SF}$ and $\mathrm{ClO}$ radicals have been carried out using the recently developed partial triples correction to Fock-space multi-reference coupled cluster method. Theoretical calculation of the doublet $\mathrm{SF}$ and $\mathrm{ClO}$ radicals is useful due to their importance in atmospheric chemistry. The dipole moments of these radicals are extremely sensitive to correlation effects. A brief insight to the way the triples correction has been implemented is presented. We compare the results obtained from our analytic response treatment with that of restricted open Hartree-Fock (ROHF) calculations. Results are presented for both relaxed and non-relaxed approach in the ROHF method. Results suggest the importance of triples corrections. The effects of orbital relaxation are also analysed from the results.
\end{abstract}

Keywords. Fock-space; Lagrangian; triples; dipole moment.

\section{Introduction}

The last couple of decades have seen a tremendous development in the field of $a b$ initio many body methods, for study of molecular electronic structure and properties. Among the available methods, coupled cluster (CC) theory ${ }^{1,2}$ has emerged as the most promising tool for electronic structure calculations. The method is noted for inclusion of size-extensivity and treatment of electron correlation in an efficient manner. Single reference coupled cluster (SRCC) $)^{3-5}$ method has been widely used for energy, molecular properties and potential energy surface calculations. ${ }^{6-16}$ Exponential wave operator introduces the dynamic correlation, in an approximate manner. Even though single reference coupled cluster singles and doubles (SR-CCSD) approximation had been well implemented for energy calculations, the inclusion of triples ${ }^{17-23}$ is often important, as was evidenced by its comparison with full CI. ${ }^{17}$ It has been shown ${ }^{19}$ that the connected part of triples, i.e., $T_{3}$ is far more important than the disconnected triple excitations like $T_{1} T_{2}$ and $\frac{1}{3 !} T_{1}^{3}$. The connected part of triples, contributes to the energy from fourth order onwards. Due to its expensive nature, in terms of computational time, a full inclusion of triples is not sought after, even

\footnotetext{
\#Dedicated to Prof. N Sathyamurthy on his 60th birthday

*For correspondence
}

though it has been pursued by Bartlett et al. ${ }^{20}$ for energy calculation. The non-iterative, partial triples correction provides an acceptable accuracy with much less computational time. Quadruple level corrections in the single reference context have also been pursued by Stanton et al. ${ }^{24}$ and Bartlett et al. ${ }^{22}$

A computational framework to analytically calculate various properties in SRCC theory was first outlined by Monkhorst ${ }^{25}$ using response approach. Since SRCC was formulated in a non-variational manner, it did not have the advantages of generalized HellmannFeynmann theorem nor the $(2 n+1)$ rule of variational theories. $^{26}$ Bartlett and co-workers ${ }^{5,6,11}$ took a step towards eliminating this apparent disadvantage using the idea of algebraic Z-vector technique introduced by Handy and Schaefer. ${ }^{27}$ But determining higher order properties using the $\mathrm{Z}$-vector technique is cumbersome. Jorgensen and co-workers, ${ }^{12}$ developed an alternative formulation of SRCC derivatives which automatically incorporates the benefits of Z-vector technique to all orders. This approach, also known as the constrained variation approach (CVA), involves construction of a functional with undetermined Lagrange multipliers.

Inspite of the above mentioned developments in SRCC, it fails to provide satisfactory physical description in cases where more than one configuration contributes dominantly to give the exact wave function (quasi-degeneracy). Such cases may arise while dealing with bond-breaking or bond-stretching. The use of a linear operator, in restricted open-shell based coupled 
cluster method to take care of such quasi-degenerate states, was implemented by Medved and co-workers. ${ }^{28}$ Solving the SRCC equations involving highly excited clusters, was another way to tackle this problem. ${ }^{29} \mathrm{In}$ this regard, inclusion of selected triples and quadruples $^{30}$ in SRCC proved efficient. However, due to the enormous computational effort involved, the inclusion of triples and quadruples are not practically feasible for large scale applications. From a physical point of view, a more satisfactory description starts with a multireference description of what is known as a reference or model space and subsequent use of an exponential wave operator. This class of methods is known as multi reference coupled cluster (MRCC) methods. The reference space takes into account the important nondynamical correlation effects. Among the various available MRCC methods, the effective Hamiltonian based method $^{31-33}$ which finds multiple roots via diagonalization of the effective Hamiltonian of the entire model space has been well explored. Within this class, two different approaches are available, namely, the Hilbertspace (HS-MRCC) ${ }^{34-36}$ approach and the Fock-space (FS-MRCC) approach. ${ }^{37-43}$ The Hilbert-space approach is based on a state universal wave operator and is best applied for handling potential energy surfaces ${ }^{44}$ (PES), also cases involving curve crossing. Mukherjee and coworkers ${ }^{45,46}$ have developed the state selective MRCC method, which has proved to be more attractive for PES. On the other hand, FSMRCC as formulated by, Kutzelnigg, ${ }^{37}$ Mukherjee ${ }^{38-40}$ and Lindgren, ${ }^{41}$ is suitable for difference energy calculations. It was applied to atoms and molecules by Kaldor et al. ${ }^{42}$ and Pal et al. ${ }^{43}$ respectively. FSMRCC approach is based on a valence universal wave operator and assumes a common vacuum to describe various states. Classifying a subset of holes and particles as active orbitals, the model space determinants are sorted into different active holeparticle sector by the addition and/or removal of electrons from the reference wave function. FSMRCC has the ability to correlate efficiently model spaces having different number of electrons, thus describing ionization potentials (IPs), electron affinities (EAs) and excitation energies (EEs) directly, where the results obtained from EE can provide a way to obtain excited states PESs too. ${ }^{47}$

Apart from these MRCC approaches, the equationof-motion (EOM) $\mathrm{CC}^{48-53}$ method and linear response $\mathrm{CC},{ }^{54,55}$ built upon a combination of linear and exponential excitation operator can also be used to handle quasi-degenerate states. The development and implementation of direct energy methods based on the CC formalism was initiated from a time-dependent linear response framework by Monkhorst. ${ }^{25}$ The EOM-CC method has been well developed for ionized, ${ }^{50,51}$ electron attached ${ }^{52}$ and excited state problems. ${ }^{48}$ Nooijen and co-workers, ${ }^{56}$ developed the similarity transformed EOMCC (STEOMCC). Spin-flip EOMCC (SF-EOMCC) ${ }^{57}$ method is another clever way to bring in the flavour of multi-reference states.

Obtaining molecular properties using effective Hamiltonian based MRCC theories was initiated by Pal. ${ }^{58,59}$ Specific expressions were obtained for one valence-hole, one valence-particle and hole-particle sectors of FSMRCC theory. Computation of first-order molecular properties, such as dipole moments, was carried out by Pal et al. ${ }^{60}$ and Ajitha and $\mathrm{Pal}^{61}$ extended the formulation to enable calculation of frequency dependent properties. Shamasunder and $\mathrm{Pal}^{62,63}$ extended the idea of Lagrange multiplier and developed the response approach within the MRCC framework. The constrained variation approach within the FSMRCC method was first formulated within the CCSD approximation. It was successfully implemented for calculation of excited state properties of small molecules and radicals. ${ }^{64-66}$ Recently, Pal and co-workers ${ }^{67}$ have developed and implemented the FSMRCC response approach for magnetic property calculations.

In the linear response (LR) CC formalism, Jorgensen et al. ${ }^{68}$ pursued response theory for molecular properties. Stanton ${ }^{69}$ proposed the theory for analytic derivatives in EOMCC method. Stanton and Gauss ${ }^{70}$ carried out the implementation of the same. EOMCC property evaluation and transition probabilities between electronic states were done by Bartlett et al. ${ }^{71}$ Gradient calculations were done by Nooijen and co-workers ${ }^{72}$ in STEOMCC using the Lagrange multipliers. In the SF-EOMCC ${ }^{73}$ method, calculation of analytic gradients at the singles and doubles truncation have been carried out.

In order to increase the accuracy of molecular properties, inclusion of the effect of triples is necessary. Iterative and non-iterative triples inclusion for energy calculations, in EOMCC ${ }^{74-78}$ and stateselective approaches ${ }^{79,80}$ have been attempted. Gauss and Stanton ${ }^{81}$ introduced perturbative triples correction to EOM-IP, while Manohar et al. ${ }^{82}$ applied the same to EOM-EA. A non-iterative triples correction to spin-flip EOMCC for excitation energies have been employed by Krylov et al. ${ }^{83}$ and Piecuch et al. ${ }^{84}$ implemented the non-iterative energy corrections to method of moments coupled cluster (MMCC) for excitation energy. Analytic derivatives at the CCSD approximation with various levels of triples inclusion has been analysed. ${ }^{85}$ Implementation of analytical gradients, for the CCSDT model was pursued by Gauss et al. ${ }^{86}$ Recently, Pal and co-workers have implemented the partial 
triples to calculate first order molecular properties in FSMRCC. ${ }^{87}$ Since the triples were added on the basis of perturbative order, hence it does not guarantee that the inclusion of triples will improve the molecular properties towards the full $\mathrm{CI}$ value.

In this paper, we will analyse the effect and importance of triples correction on the first order response properties (dipole moment) of $\mathrm{SF}$ and $\mathrm{ClO}$ radicals computed using the Fock-space multi-reference coupled cluster theory. The results obtained from FSMRCC are compared with the finite difference ROHF- coupledcluster calculations. The effects of relaxation are also analysed. Both these radicals play an important role in atmospheric chemistry. Hence a detailed knowledge regarding their molecular properties is necessary.

In section 2, a brief review of the constrained variation approach in FSMRCC method is outlined. In section 3, a detailed implementation of the partial triples correction is described. Section 4 comprises the computational details. The results and discussions are presented in section 5, followed by the conclusions in section 6 .

\section{Brief resumé of FSMRCC}

The FSMRCC ${ }^{37,39-41,43}$ and the Langrangian formulation ${ }^{63-65}$ within the same have been well described. A brief review of the same is given in this paper. The FSMRCC approach is based on a common vacuum concept. The Hartree-Fock solution for the closed shell Nelectron ground state is chosen as the vacuum. Particles and holes are defined with respect to this vacuum. Furthermore, these are subdivided into active and inactive holes and particles. The number of active particles and active holes in a function is represented by the superscript. Thus, a general model space, containing $m$-active particles and $n$-active holes is represented as:

$$
\left|\Psi_{(0) \mu}^{(m, n)}\right\rangle=\sum_{i} C_{\mu i}^{(m, n)}\left|\Phi_{i}^{(m, n)}\right\rangle,
$$

where, $C_{\mu i}^{(m, n)}$ are the model space or combination coefficients of $\Phi_{i}$. The total or the correlated wave function for the $\mu^{\text {th }}$ state is given by,

$$
\left|\Psi_{\mu}^{(m, n)}\right\rangle=\Omega\left|\Psi_{(0) \mu}^{(m, n)}\right\rangle .
$$

Here $\Omega$ is the universal wave operator, which generate states by its action on the reference wave function. The generated states are such that they satisfy the Bloch Equation. $\Omega$ is denoted as:

$$
\Omega=\left\{e^{\tilde{T}(m, n)}\right\},
$$

where, the parenthesis denotes normal ordering of operators. Lindgren had introduced the normal ordered operator. ${ }^{15}$ The cluster operator $\tilde{T}^{(m, n)}$ is expressed as:

$$
\tilde{T}^{(m, n)}=\sum_{k=0}^{m} \sum_{l=0}^{n} T^{(k, l)}
$$

where $T^{(k, l)}$ can create particles and holes in addition to destroying exactly $k$ active particles and $l$ active holes. In the Fock-space approach, $\tilde{T}^{(m, n)}$ contains all the lower valence amplitudes thus giving additional flexibility to the theory. To calculate ionization potential, we define our system as a specific problem of $(0,1)$ sector, i.e. zero active particle and one active hole sector. The Schrödinger equation for quasi-degenerate states is given by,

$$
H\left|\Psi_{\mu}^{(0,1)}\right\rangle=E_{\mu}\left|\Psi_{\mu}^{(0,1)}\right\rangle
$$

which gives,

$$
H \Omega\left(\sum_{i} C_{\mu i}^{(0,1)}\left|\Phi_{i}^{(0,1)}\right\rangle\right)=E_{\mu} \Omega\left(\sum_{i} C_{\mu i}^{(0,1)}\left|\Phi_{i}^{(0,1)}\right\rangle\right) \text {. }
$$

An effective Hamiltonian $\left(H_{e f f}\right)$ is defined through the Bloch equations:

$$
\begin{aligned}
& P^{(0,1)}\left(H \Omega-\Omega H_{e f f}^{(0,1)}\right) P^{(0,1)}=0 \\
& Q^{(0,1)}\left(H \Omega-\Omega H_{e f f}^{(0,1)}\right) P^{(0,1)}=0,
\end{aligned}
$$

where $P^{(0,1)}$ is the Projection operator for the model space, defined as:

$$
P^{(0,1)}=\sum_{i}\left|\Phi_{i}^{(0,1)}\right\rangle\left\langle\Phi_{i}^{(0,1)}\right| .
$$

The complimentary space $Q^{(0,1)}$ is defined in the following manner

$$
Q^{(0,1)}=1-P^{(0,1)} .
$$

The $T$ amplitude equations are solved by the aid of subsystem embedding condition (SEC). The ground state coupled-cluster amplitudes $T^{(0,0)}$ are solved followed by the equation for $T^{(0,1)}$ amplitudes. Normal ordering in the wave operator $\Omega$ ensures that the $T^{(0,1)}$ amplitudes do not occur while solving for the $(0,0)$ sector i.e. ground state. While in the $(0,1)$ valence sector, the $T^{(0,0)}$ amplitudes occur as constant entities. This is known as SEC. Thus, SEC and normal ordering ensures a hierarchical decoupling of the various Fock-space sectors.

A satisfactory formulation of analytic derivatives in the context of effective Hamiltonian version of MRCC methods could only be obtained using the constrained variational approach (CVA). Szalay ${ }^{88}$ had been the first 
to apply CVA in FSMRCC context and analyse the functional for gradient calculations. Based on the Langranges method of undetermined multipliers, in CVA, the Langragian is constructed for a specific root of the effective Hamiltonian. Pal and co-workers formulated the CVA-FSMRCC technique for specific root of the effective Hamiltonian which is applicable for general incomplete model spaces (IMS). The energy of a specific state of the ionized system is given by,

$$
E_{\mu}=\sum_{i j} \tilde{C}_{\mu i}^{(0,1)}\left(H_{e f f}\right)_{i j}^{(0,1)} C_{j \mu}^{(0,1)} .
$$

The Lagrangian is constructed to minimize the energy expression given above, with the constraint that the MRCC equations (i.e., the Bloch Equations) are satisfied for the specific state ' $\mu$ '

$$
\begin{aligned}
\Im= & \sum_{i j} \tilde{C}_{\mu i}^{(0,1)}\left(H_{e f f}\right)_{i j}^{(0,1)} C_{j \mu}^{(0,1)} \\
& +\sum_{j i} \Lambda_{j i}^{(0,1)}\left\langle\phi_{j}^{(0,1)}\left|\left(H \Omega-\Omega H_{e f f}\right)\right| \phi_{i}^{(0,1)}\right\rangle \\
& +\sum_{\alpha} \sum_{i} \Lambda_{\alpha i}^{(0,1)}\left\langle\phi_{\alpha}^{(0,1)}\left|\left(H \Omega-\Omega H_{e f f}\right)\right| \phi_{i}^{(0,1)}\right\rangle \\
& +\sum_{j i} \Lambda_{j i}^{(0,0)}\left\langle\phi_{j}^{(0,0)}|H \Omega| \phi_{i}^{(0,0)}\right\rangle \\
& +\sum_{\alpha} \sum_{i} \Lambda_{\alpha i}^{(0,0)}\left\langle\phi_{\alpha}^{(0,0)}|H \Omega| \phi_{i}^{(0,0)}\right\rangle \\
& -E_{\mu}\left(\sum_{i j} \tilde{C}_{\mu i}^{(0,1)} C_{j \mu}^{(0,1)}-1\right)
\end{aligned}
$$

where, $\phi_{i}^{(0,1)}, \phi_{j}^{(0,1)}, \phi_{i}^{(0,0)}$ and $\phi_{j}^{(0,0)}$ are the model space functions, i.e. functions in $P$ space. On the other hand, $\phi_{\alpha}^{(0,1)}$ and $\phi_{\alpha}^{(0,0)}$ are the functions in the complimentary space, i.e. in $Q$ space. The $\Lambda$ in the above equation are the Langrange multipliers. $\Lambda_{j i}^{(0,1)}$ and $\Lambda_{j i}^{(0,0)}$ are defined within the $P-P$ space, whereas $\Lambda_{\alpha i}^{(0,1)}$ and $\Lambda_{\alpha i}^{(0,0)}$ are defined in the $P-Q$ space for $(0,1)$ and $(0,0)$ sectors, respectively. But in the case of complete model space (CMS), the effective Hamiltonian has an explicit expression in terms of the cluster operators, as a result of which, the closed part in the Lagrange multiplier vanishes, reducing the above equation to

$$
\begin{aligned}
\Im= & \sum_{i j} \tilde{C}_{\mu i}^{(0,1)}\left(H_{e f f}\right)_{i j}^{(0,1)} C_{j \mu}^{(0,1)} \\
& +\sum_{\alpha} \sum_{i} \Lambda_{\alpha}^{(0,1)}\left\langle\phi_{\alpha}^{(0,1)}\left|\left(H \Omega-\Omega H_{e f f}\right)\right| \phi_{i}^{(0,1)}\right\rangle \\
& +\sum_{\alpha} \sum_{i} \Lambda_{\alpha i}^{(0,1)}\left\langle\phi_{\alpha}^{(0,0)}|H \Omega| \phi_{i}^{(0,0)}\right\rangle \\
& -E_{\mu}\left(\sum_{i j} \tilde{C}_{\mu i}^{(0,1)} C_{j \mu}^{(0,1)}-1\right) .
\end{aligned}
$$

Differentiation of the above equation with respect to $\Lambda$ gives the expression for cluster amplitudes, i.e. the Bloch equations. Differentiating the same with respect to the $T$ amplitudes will furnish equations for Langrange multipliers $\Lambda$. The equation for the cluster amplitudes is decoupled from the $\Lambda$-amplitude equations. The $\Lambda$ equations are however, coupled with those of the $T$ amplitudes. In the presence of an external field, the Lagrangian and the parameters $H_{e f f}, C, \tilde{C}, E, \Omega$ and $\Lambda$ all become perturbation dependent. Differentiation of the Lagrangian with respect to the unperturbed cluster amplitudes, generate equations for the Lagrange multipliers, whereas differentiation of the same with respect to the unperturbed Lagrange multipliers will furnish equations for the cluster amplitudes. The energy derivatives follow the $(2 n+1)$ rule with respect to the cluster amplitudes and $(2 n+2)$ rule with respect to the $\Lambda$-amplitudes (Lagrange multipliers). A $(2 n+1)$ rule holds true for the eigen-vectors $\tilde{C}^{(0,1)}$ and $C^{(0,1)}$ for the evaluation of energy derivatives. It is worth mentioning that the coupling within the $\Lambda$-amplitudes in various valence sectors is exactly opposite to that of SEC. Thus, we first solve the $\Lambda$-amplitudes for the highest valence sector and then move towards the lowest valence sector.

\section{Method of triples implementation}

Several schemes for the inclusion of triples are available in the literature. In this particular section, we describe the scheme that we have implemented in our triples formulation. This section will deal with the implementation of the triples to the dipole moment. We first discuss the implementation of the non-iterative triples in $T$ and then in $\Lambda$-amplitudes to the dipole moment in FSMRCC response.

We start with the specific way the triples correction have been added to the SRCC equations, i.e., the $(0,0)$ sector.

Canonical orbitals have been used for our calculation, i.e., the orbitals have not been allowed to relax or change with the perturbation. Hence, this is a nonrelaxed approach. First the $T_{1}^{(0,0)}$ and the $T_{2}^{(0,0)}$ amplitudes are solved in a completely iterative manner, which is the general CCSD approximation. Using these generated $T_{1}^{(0,0)}$ and $T_{2}^{(0,0)}$ amplitudes, $T_{3}^{(0,0)}$ is calculated in a non-iterative manner from $V T_{2}^{(0,0)}$ and $V T_{2}^{(0,0)} T_{2}^{(0,0)}$. The contribution made by $V T_{2}^{(0,0)}$ and $V T_{2}^{(0,0)} T_{2}^{(0,0)}$ is at the second and third order of perturbation, respectively. The CCSD equations are updated by including the $V T_{3}^{(0,0)}$ term. Inclusion of $V T_{3}^{(0,0)}$ in the $T_{3}^{(0,0)}$ equation will convert the entire method 
iterative. Hence, the $V T_{3}^{(0,0)}$ is excluded from the $T_{3}^{(0,0)}$ equations in this particular scheme of triples correction.

Now, the effective Hamiltonian for the one valence sector, under this approximation is:

$$
\begin{aligned}
H_{e f f}=P^{(0,1)} & \left(\bar{F}+\bar{F} T_{1}^{(0,1)}+\bar{V} T_{2}^{(0,1)}\right. \\
& \left.+\bar{F} T_{2}^{(0,1)}+\bar{V} T_{3}^{(0,1)}\right) P^{(0,1)} .
\end{aligned}
$$

It can be seen easily that $\bar{W}$ cannot contribute to $H_{\text {eff }}$ and hence is not included. $V T_{3}^{(0,1)}$ is the only term that contributes to the singles and doubles amplitude equation along with $H_{\text {eff }}$.

The Fock-space Bloch equations, for the $T_{1}^{(0,1)}, T_{2}^{(0,1)}$ and $T_{3}^{(0,1)}$ amplitudes are given by,

$$
\begin{gathered}
Q_{1}^{(0,1)}\left(\bar{F}+\bar{F} T_{1}^{(0,1)}+\bar{V} T_{2}^{(0,1)}+\bar{F} T_{2}^{(0,1)}\right. \\
\left.+\bar{V} T_{3}^{(0,1)}-T_{1}^{(0,1)} H_{e f f}\right) P^{(0,1)}=0 \\
Q_{2}^{(0,1)}\left(\bar{V}+\bar{F} T_{2}^{(0,1)}+\bar{V} T_{1}^{(0,1)}+\bar{V} T_{2}^{(0,1)}+\bar{W} T_{2}^{(0,1)}\right. \\
\left.+\bar{V} T_{3}^{(0,1)}+\bar{F} T_{3}^{(0,1)}-T_{2}^{(0,1)} H_{e f f}\right) P^{(0,1)}=0 \\
Q_{3}^{(0,1)}\left(\bar{W}+\bar{W} T_{2}^{(0,1)}+\bar{F} T_{3}^{(0,1)}+\bar{V} T_{2}^{(0,1)}\right. \\
\left.-T_{3}^{(0,1)} H_{e f f}\right) P^{(0,1)}=0 .
\end{gathered}
$$

Since, we want to be accurate only up to third order, the term that contributes to $T_{3}^{(0,1)} H_{\text {eff }}$ is only $\bar{F} T_{3}^{(0,1)}$. The equations 14 and 15 are first solved fully, excluding those terms which involve $T_{3}^{(0,1)}$ amplitude. This is the normal CCSD approximation. Using the known amplitudes equation 16 is solved in a non-iterative manner. After solving for $T_{3}^{(0,1)}$ equation, equations 14 and 15 are solved in an iterative manner once again. This brings in the effect of $T_{3}^{(0,1)}$ via $\bar{V} T_{3}^{(0,1)}$ and $\bar{F} T_{3}^{(0,1)}$.

After the completion of the triples correction to energy, we examine the way the triples correction is added to the $\Lambda$ amplitude and finally to the dipole moment. While solving the $\Lambda$ amplitude, we need to first solve for the $(0,1)$ sector and then the $(0,0)$ sector, which is the opposite way to solve these equations as compared to the $T$ amplitudes due to reverse decoupling in the $\Lambda$-equations. First the $\Lambda$-amplitudes are solved iteratively within the CCSD approximation for both $(0,1)$ and $(0,0)$ sector. With those generated $\Lambda$ amplitudes, the Lagrangian for the triples correction is constructed. The triples corrected Lagrangian is given by,

$$
\begin{aligned}
\Im= & S D+\bar{V} T_{3}^{(0,1)} C \tilde{C}+\Lambda_{3}^{(0,1)} \bar{V} T_{2}^{(0,1)} \\
& +\Lambda_{3}^{(0,1)} \bar{W} T_{2}^{(0,1)}+\Lambda_{3}^{(0,1)} \bar{F} T_{3}^{(0,1)} \\
& +\Lambda_{2}^{(0,1)} \bar{V} T_{3}^{(0,1)}+\Lambda_{2}^{(0,1)} \bar{F} T_{3}^{(0,1)} \\
& -\Lambda_{2}^{(0,1)} T_{2}^{(0,1)} \bar{V} T_{3}^{(0,1)}+\Lambda_{1}^{(0,1)} \bar{V} T_{3}^{(0,1)} \Lambda_{3}^{(0,0)} V T_{2}^{(0,0)} \\
& +\Lambda_{2}^{(0,0)} V T_{3}^{(0,0)}+\Lambda_{1}^{(0,0)} V T_{3}^{(0,0)}+\Lambda_{3}^{(0,0)} F T_{3}^{(0,0)} \\
& +\Lambda_{3}^{(0,1)} \bar{W} T_{2}^{(0,0)}+\Lambda_{3}^{(0,1)} V T_{3}^{(0,0)}+\Lambda_{3}^{(0,1)} V T_{2}^{(0,0)}
\end{aligned}
$$

where, $\Lambda_{3}$ represents the Langrangian for triples, $\tilde{C}$ and $C$ are the left and right eigenvectors of the $H_{\text {eff }}$. The Lagrangian in equation 17 is differentiated with respect to $T_{3}^{(0,1)}$ to get the $\Lambda_{3}^{(0,1)}$ equation. The equation comprising $\Lambda_{3}^{(0,1)}$ amplitude is:

$$
\begin{aligned}
& \left\langle P^{(0,1)}\right| \bar{V} C \tilde{C}+\Lambda_{3}^{(0,1)} \bar{F}+\Lambda_{2}^{(0,1)} \bar{V}-\Lambda_{2}^{(0,1)} T_{2}^{(0,1)} \bar{V} \\
& +\Lambda_{1}^{(0,1)} \bar{V}\left|Q^{(0,1)}\right\rangle=0 .
\end{aligned}
$$

The Lagrangian in equation 17 is differentiated with respect to $T_{2}^{(0,1)}$ to get the $\Lambda_{2}^{(0,1)}$ equation. The $\Lambda_{2}^{(0,1)}$ equation is given below:

$$
S D+\left\langle P^{(0,1)}\left|\Lambda_{3}^{(0,1)} \bar{V}+\Lambda_{3}^{(0,1)} \bar{W}\right| Q^{(0,1)}\right\rangle=0 .
$$

Equation 18 is solved in a non-iterative manner to obtain $\Lambda_{3}^{(0,1)}$. Only the connected terms in $\Lambda_{3}^{(0,1)}$ amplitude equation are considered here. $\bar{V} C \tilde{C}, \Lambda_{3}^{(0,1)} \bar{F}$, $\Lambda_{2}^{(0,1)} \bar{V}, \Lambda_{2}^{(0,1)} T_{2}^{(0,1)} \bar{V}$ and $\Lambda_{1}^{(0,1)} \bar{V}$ are the terms contributing to $\Lambda_{3}^{(0,1)}$. Their contribution occurs in the first order, second order, second order, third order and second order respectively. After obtaining $\Lambda_{3}^{(0,1)}$ its effect on $\Lambda_{2}^{(0,1)}$ is felt through the third order terms $\Lambda_{3}^{(0,1)}$ and $\Lambda_{3}^{(0,1)} \bar{W}$. The $\Lambda_{2}^{(0,1)}$ amplitude equation is solved by taking into account the $\Lambda_{3}^{(0,1)}$ terms calculated previously.

In order to solve the Lagrange multipliers for the $(0,0)$ sector, we have to solve the $\Lambda_{3}^{(0,0)}$ equations first. Following the footsteps of the $(0,1)$ sector, here too, the $\Lambda_{3}^{(0,0)}$ equation is obtained by differentiating the Lagrangian in equation 17 with respect to $T_{3}^{(0,0)}$. The terms which appear after differentiation is given below:

$$
\left\langle P^{(0,0)}\left|\Lambda_{3}^{(0,0)} F+\Lambda_{2}^{(0,0)} V+\Lambda_{1}^{(0,0)} V+\Lambda_{3}^{(0,1)} V\right| Q^{(0,0)}\right\rangle=0 .
$$

Similar to its treatment in the $(0,1)$ sector, the equation for $\Lambda_{2}^{(0.0)}$ is obtained by differentiating equation 17 with 
respect to $T_{2}^{(0,0)}$. The $\Lambda_{2}^{(0,0)}$ equation with the triples correction is:

$S D+\left\langle P^{(0,0)}\left|\Lambda_{3}^{(0,0)} V+\Lambda_{3}^{(0,1)} V+\Lambda_{3}^{(0,0)} V T_{2}^{(0,0)}\right| Q^{(0,0)}\right\rangle=0$

$\Lambda_{3}^{(0,0)}$ is obtained by solving equation 20 in a noniterative fashion. The terms contributing to $\Lambda_{3}^{(0,0)}$ are $\Lambda_{3}^{(0,0)} F, \Lambda_{2}^{(0,0)} V, \Lambda_{1}^{(0,0)} V$ and $\Lambda_{3}^{(0,1)} V$. They contribute at the second order, second order, third order and third order, respectively. Due to reverse decoupling, the $\Lambda^{(0,1)}$ containing terms i.e., $\Lambda_{3}^{(0,1)} V T_{2}^{(0,0)}$ and $\Lambda_{3}^{(0,1)} V T_{3}^{(0,0)}$ appear in $\Lambda^{(0,0)}$. The effect of $\Lambda_{3}^{(0,0)}$ on $\Lambda_{2}^{(0,0)}$ is obtained via the third order terms $\Lambda_{3}^{(0,0)} V, \Lambda_{3}^{(0,1)} V$ and $\Lambda_{3}^{(0,0)} V T_{2}^{(0,0)}$. Taking into account all the $\Lambda_{3}^{(0,0)}$ containing terms, equation 21 is solved. The triples contribution to $E^{(1)}$ is given below:

$$
E_{\text {triples }}^{(1)}=\Lambda_{2}^{(0,1)} \hat{O} T_{3}^{(0,1)}+\Lambda_{2}^{(0,0)} \hat{O} T_{3}^{(0,0)}
$$

where, $\hat{O}$ is the explicit derivative of the Hamiltonian with respect to the external field.

In the evaluation of the final dipole moments for the $(0,0)$ as well as the $(0,1)$ sector, the final triples corrected $\Lambda$ and $T$-amplitudes are used. The third order terms which appear in the dipole moment equation are, $\Lambda_{2}^{(0,1)} \hat{O} T_{3}^{(0,1)}$ and $\Lambda_{2}^{(0,0)} \hat{O} T_{3}^{(0,0)}$. The $V T_{2}^{(0,0)} T_{2}^{(0,0)}$ term which is present in the $T_{3}^{(0,0)}$ equation, will have a higher order effect on the dipole moment. So, the final dipole moment is corrected at least up to third order in triples.

\section{Computational details}

The importance of $\mathrm{SF}$ and $\mathrm{ClO}$ radicals are seen in atmospheric chemistry. Hence, a detailed theoretical knowledge regarding their inherent properties is required.

The ground state electronic structure of $\mathrm{ClO}$ and SF is ${ }^{2} \Pi$, as is expected from simple molecular orbital consideration. The $\mathrm{ClO}$ and $\mathrm{SF}$ anions are closed shell anions. Hence, they are chosen as the restricted Hartree-Fock (RHF) vacuum for our calculations. The highest occupied molecular orbital (HOMO) of both $\mathrm{CIO}^{-}$and $\mathrm{SF}^{-}$are two-fold degenerate in nature. So the HOMO's are chosen as active holes in the Fockspace $(0,1)$ sector. The removal of an electron from any one of these orbitals leads to a degenerate doublet radical.

The restricted open Hartree-Fock (ROHF) based couple-cluster calculations are done using the ACES II package. ${ }^{89}$ The ROHF based results (both relaxed and non-relaxed) presented in this paper have been calculated through finite difference of energy points, employing field displacements of \pm 0.001 a.u. for dipole moment computations. Both relaxed and nonrelaxed approaches have been used for ROHF based calculations.

\section{Results and discussions}

In an earlier paper, the triples corrected code was tested and pilot results were obtained for quite a few radicals. ${ }^{87}$ In this paper, $\mathrm{ClO}$ and $\mathrm{SF}$ radicals are studied using this triples corrected FSMRCC response theory. We compare our results with the ROHF based coupledcluster finite-field method.

\subsection{ClO radical}

The importance of halogen monoxide in atmospheric ozone depletion chemistry has motivated a detailed study, both experimentally and theoretically. The chlorine monoxide radical is an important participant in the catalytic destruction of ozone in the earth's stratosphere. The chlorine monoxide is a particularly interesting molecule in that it possesses a ${ }^{2} \Pi$ ground state with an inverted spin doublet. ${ }^{90}$ The ${ }^{2} \Pi_{3 / 2}$ level is the lowest lying electronic state for this radical. Even though the $\mathrm{ClO}$ radical has been studied extensively in terms of experimental methods, theoretical calculations are still lacking. The reason behind this could be that the $\mathrm{ClO}$ radical is considered to be a 'difficult' case. ${ }^{91}$ Petersson et al. ${ }^{91(\mathrm{~b})}$ stated that the ${ }^{2} \Pi$ state of $\mathrm{ClO}$ is not very well described by a single-reference configuration. Thus, MRCC description seems to be appropriate for this study.

The calculations have been presented with the $\mathrm{Cl}-\mathrm{O}$ bond length as 3.1898 Bohr. The RHF of chlorine monoxide anion is chosen as a vacuum and the radical has been described as one hole in Fock-space description. Table 1 represents the dipole moments of the $\mathrm{ClO}$ radical in cc-pVDZ and cc-PVTZ basis. These have been compared with the non-relaxed ROHF dipole moment values. The best MR-CI calculation led to a dipole moment value ${ }^{91(b)}$ of $1.275 \mathrm{D}$ for the ground state. But this reported value also includes an approximate correction for quadruples. Our calculation of triples correction shows a general trend towards this value. The $\Lambda$-FSMRCC result shows the convergence towards the experimental value on going from CCSD to $\operatorname{CCSD}\left(\mathrm{T}^{*}\right)$. For a better correlated basis set, i.e., cc-pVTZ, the triples correction, over the singles and 
Table 1. Dipole moment results of $\mathrm{ClO}$ radical.

\begin{tabular}{|c|c|c|c|c|c|c|}
\hline \multirow[t]{2}{*}{ Basis } & \multicolumn{2}{|c|}{$\Lambda-$ FSMRCC } & \multicolumn{2}{|c|}{$\mathrm{ROHF}^{\mathrm{a}}$ non-relaxed } & \multicolumn{2}{|c|}{$\mathrm{ROHF}^{\mathrm{a}}$ relaxed } \\
\hline & $\mathrm{CCSD}$ & $\operatorname{CCSD}\left(\mathrm{T}^{*}\right)$ & $\mathrm{CCSD}$ & $\operatorname{CCSD}(\mathrm{T})$ & $\mathrm{CCSD}$ & $\operatorname{CCSD}\left(\mathrm{T}^{*}\right)$ \\
\hline \multicolumn{7}{|c|}{ Experimental dipole: $1.2974 \mathrm{D}^{95}$} \\
\hline \multicolumn{7}{|c|}{ MR-CI dipole: $1.275 \mathrm{D}^{91(\mathrm{~b})}$} \\
\hline cc-pVDZ & 1.051 & 1.418 & 1.160 & 1.166 & 1.131 & 1.153 \\
\hline cc-pVTZ & 1.081 & 1.147 & 1.148 & 1.152 & 1.112 & 1.150 \\
\hline
\end{tabular}

All results in Debye.

Cl-O bond length is 3.1898 Bohr, ${ }^{a}$ ROHF results obtained from ACES II package ${ }^{89}$

doubles approximation is seen to improve the result, from 1.081D to $1.147 \mathrm{D}$, towards the experimental value of $1.2974 \mathrm{D}$. For the cc-pVDZ basis, the $\operatorname{CCSD}\left(\mathrm{T}^{*}\right)$ result shows a much higher correction as compared to that of cc-pVTZ basis. This is due to the fact that the cc-pVDZ basis does not include correlation effects as well as that included in the triple zeta basis. Hence, the triples correction is more for that particular basis. Even though in case of cc-pVDZ basis, the theoretical value overshoots the experimental one, the qualitative trend towards the experimental dipole moment and MR-CI is obtained. On comparison of the ROHF relaxed and nonrelaxed approach, we find that the relaxed approach predicts a lower dipole moment than the non-relaxed one. The $\Lambda$-FSMRCC method is an analytic non-relaxed based method. So, in order to have a better comparison, we have employed non-relaxed approach for the ROHF-CC calculations too. The same geometry and basis set have been used for calculating dipole moments in both the methods. The ROHF based coupled-cluster dipole moment values for both relaxed and non-relaxed cases also show an improvement towards the experimental value on including the effects of triples. Hence, we can conclude that the triples correction is essential for handling such "difficult" cases of radicals, in order to have a more accurate description of their properties.

\subsection{SF radical}

Sulphur containing molecules and radicals are very important in atmospheric chemistry, thermal chemistry, combustion chemistry and interstellar chemistry. As one of the simplest sulphur containing molecule, the SF radical is highly reactive and has been extensively studied both experimentally and theoretically. SF is one of the degradation products of $\mathrm{SF}_{6}$ formed in high voltage power systems, where it is employed as an insulator. Traces of SF are also formed when $\mathrm{SF}_{4}$ is dissociated in a shock tube at temperatures above $2000 \mathrm{~K} .{ }^{88}$

Since the SF radical is highly reactive, it is difficult to handle in the laboratory. This makes theoretical approach a useful source of information. We treat the SF radical in a similar manner as the $\mathrm{ClO}$ radical. For SF radical, we report the dipole moment calculated at the experimental ground state geometry of $r_{0}=1.600575 \AA .^{92}$

CI(SD) and CEPA-3(SD) theoretical calculations, ${ }^{93}$ done a couple of decades ago, shows the dipole moments to be 1.106 and 0.968 , respectively calculated at the S-F bond distance 3.02 Bohr (1.5981 $⿱$ ) for small basis sets. The experimental value is stated as $0.87 \mathrm{D}$ (approx). ${ }^{94}$

In table 2, we report the dipole moment values of the $\mathrm{SF}$ radical calculated at cc-pVDZ and cc-pVTZ basis

Table 2. Dipole moments of SF radical.

\begin{tabular}{|c|c|c|c|c|c|c|}
\hline \multirow[t]{2}{*}{ Basis } & \multicolumn{2}{|c|}{$\Lambda-\mathrm{FSMRCC}$} & \multicolumn{2}{|c|}{$\mathrm{ROHF}^{\mathrm{a}}$ non-relaxed } & \multicolumn{2}{|c|}{$\mathrm{ROHF}^{\mathrm{a}}$ relaxed } \\
\hline & CCSD & $\operatorname{CCSD}\left(\mathrm{T}^{*}\right)$ & CCSD & $\operatorname{CCSD}(\mathrm{T})$ & CCSD & $\operatorname{CCSD}\left(\mathrm{T}^{*}\right)$ \\
\hline \multicolumn{7}{|c|}{ Experimental dipole: $0.87 \pm 0.05 \mathrm{D}^{94}$} \\
\hline \multicolumn{7}{|c|}{$\mathrm{CI}(\mathrm{SD})=1.106 \mathrm{D}^{93}, \mathrm{CEPA}-3(\mathrm{SD})=0.968 \mathrm{D}^{93}$} \\
\hline cc-pVDZ & 1.027 & 1.127 & 1.046 & 1.049 & 1.128 & 1.062 \\
\hline cc-pVTZ & 0.892 & 1.064 & 0.895 & 0.878 & 0.895 & 0.878 \\
\hline
\end{tabular}

All results in Debye. $r_{0}=1.600575 \AA{ }^{92}$ a ROHF results obtained from ACES II package $^{89}$ 
sets. It is observed that the dipole moment value converge towards the reported experimental dipole moment value on moving from cc-pVDZ to cc-pVTZ basis. The dipole moment calculated via CCSD method in the ccpVTZ basis is already very close to the experimental value and hence the triples correction is seen to overshoot the experimental value. In both the basis set it is observed that the triples correction tends to increase the dipole moment value, as opposed to the trend observed in ROHF-based cases. The cc-pVTZ basis set improves the dipole moment value towards the experimental one. Hence the cc-pVTZ is seen to be a better basis set due to its inbuilt triple zeta correlated wave function than the cc-pVDZ basis.

The dipole moment values obtained from the $\Lambda$ FSMRCC calculations tend to move away from the experimental value. But it tends to converge towards the reported CI value. The ROHF based calculations are done in finite difference method, while the $\Lambda$ FSMRCC is an analytic approach. The difference in the non-relaxed ROHF- coupled-cluster and $\Lambda$-FSMRCC dipole moment results may have arisen due to the difference in approaches to calculate the first derivatives. The opposite trend of the triples correction as seen in the $\Lambda$-FSMRCC and ROHF-CC calculation is rather difficult to explain at the first glance. However, the way the partial triples are included in both the approaches are not exactly the same and this could have led to discrepancies in this particular case.

We have also reported relaxed finite difference calculations in ROHF. A comparison between the relaxed and non-relaxed ROHF CC calculations shows the effects of relaxation. It is also seen that in a better basis (i.e., cc-pVTZ) the calculated dipole moment in ROHF$\operatorname{CCSD}(\mathrm{T})$ approximation shows very good agreement with the experimental value.

\section{Conclusions}

In this paper, we have presented the recently implemented Lagrange based Fock-space multireference coupled cluster response approach with the inclusion of partial triples for electric properties of radicals. The dipole moment values for the $\mathrm{ClO}$ radical shows that the inclusion of partial triples i.e., $\Lambda$-FSMRCCSD(T*) converge the dipole moment values toward the experimental value, hence the inclusion of triples lead to more accurate results. Even though in the case of the cc$\mathrm{pVDZ}$ basis the triples correction goes beyond the predicted experimental value, its basic trend is in the right direction. ROHF also predicts the same trend in the triples correction. On the other hand, in case of the SF radical, the CCSD value of $0.892 \mathrm{D}$ is already in good agreement with the experimental value of $0.87 \mathrm{D}$. On including the partial triples it overestimates the dipole moment value to $1.064 \mathrm{D}$. The triples correction in the ROHF calculation is seen to match fairly well with the predicted experimental dipole moment. However, the CISD and CEPA-3(SD) results are in better agreement with that of $\Lambda$-FSMRCCSD(T*). In case of the SF radi$\mathrm{cal}$, it is seen that the ROHF and FSMRCC triples addition follow opposite trend. A probable reason could be the way, the effect of triples have been implemented in both the methods and the different manner in which the dynamical correlation has been taken into account.

\section{Acknowledgements}

The authors acknowledge the facilities provided by the Centre of Excellence in Scientific Computing present at NCL. Both the authors SP and DB acknowledge the grant sanctioned from the Department of Science and Technology (DST) through JC Bose Fellowship project for financial support. One of the authors LR would like to thank the Council of Scientific and Industrial Research (CSIR) for a Senior Research Fellowship.

\section{References}

1. (a) Cizek J 1969 Adv. Chem. Phys. 14 35; (b) Cizek J 1966 J. Chem. Phys. 454256

2. Paldus J, Cizek J and Shavitt I 1972 Phys. Rev. A: At. Mol. Opt. Phys. 550

3. Bartlett R J 1981 Аnnu. Rev. Phys. Chem. 32359

4. (a) Coester F 1958 Nucl. Phys. 7 421; (b) Coester F and Kümmel H 1960 Nucl. Phys. 17477

5. (a) Bartlett R J 1995 In Modern electronic structure theory (ed.) D R Yarkony (Singapore: World Scientific Publishing Co. Pte. Ltd.) Vol. 2, p. 1047; (b) Bartlett R J 1986 Geometrical derivatives of energy surfaces and molecular properties (eds) P Jorgensen and J Simons Reidel, (Dordrecht, The Netherlands) 3561

6. Adamowicz L, Laidig W D and Bartlett R J 1984 Int. J. Quantum Chem. 26245

7. Scheiner A C, Scuseria G E, Lee T J, Rice J E and Schaefer H F III 1987 J. Chem. Phys. 875361

8. Wang F and Gauss J 2008 J. Chem. Phys. 129174110

9. Kállay M, Gauss J and Szalay P G 2003 J. Chem. Phys. 1192991

10. Monkhorst H 1977 Int. J. Quantum Chem. 12421

11. Salter E A, Trucks G W and Bartlett R J 1989 J. Chem. Phys. 901752

12. (a) Helgaker $\mathrm{T}$ and Jørgensen P 1988 Adv. Quantum Chem. 19 183; (b) Helgaker T and Jørgensen P 1988 J. Chem. Phys. 89 1560; (c) Koch H, Jensen H J A, Jørgensen P, Helgaker T, Scuseria G E and Schaefer H F III 1990 J. Chem. Phys. 924924

13. Christiansen O, Koch H and Jørgensen P 1995 Chem. Phys. Lett. 243409 
14. Lindgren I 1978 Int. J. Quantum Chem. S12 33

15. Koch H, Christiansen O, Jørgensen P, de Meras A M S and Helgaker T 1997 J. Chem. Phys. 1061808

16. Medved' M, Urban M and Noga J 1997 Theor. Chem. Acc. 9875

17. (a) Lee Y S, Kucharski S A and Bartlett R J 1984 J. Chem. Phys. 81 5906; (b) Noga J, Bartlett R J and Urban M 1987 Chem. Phys. Lett. 134126

18. Scuseria G E and Schaefer H F III 1988 Chem. Phys. Lett. 14623

19. Noga J and Bartlett R J 1987 J. Chem. Phys. 867041

20. Kucharski S A and Bartlett R J 1998 J. Chem. Phys. 108 5243

21. Raghavachari K, Trucks G W, Polpe J A and HeadGordon M 1989 Chem. Phys. Lett. 157479

22. Bartlett R J, Watts J D, Kucharski S A and Noga J 1990 Chem. Phys. Lett. 165513

23. Urban M, Noga J, Cole S J and Bartlett R J $1985 \mathrm{~J}$. Chem. Phys. 834041

24. Bomble Y J, Stanton J, Kallay M and Gauss J 2005 J. Chem. Phys. 123054101

25. Monkhorst H J 1977 Int. J. Quantum Chem. S11 421

26. Epstein S T 1974 The variation principle in quantum chemistry (New York: Academic)

27. Handy N C and Schaefer H F III 1984 J. Chem. Phys. 81 5031

28. Medved' M, Urban M, Kello V and Diercksen G H F 2001 J. Mol. Struct. Theochem. 547219

29. Ghosh K B, Piecuch P, Pal S and Adamowicz L 1996 J. Chem. Phys. 1046582

30. (a) Piecuch P and Adamowicz L 1994 J. Chem. Phys. 100 5857; (b) Oliphant N and Adamowicz L $1991 \mathrm{~J}$. Chem. Phys. 94 1129; (c) Oliphant N and Adamowicz L 1992 J. Chem. Phys. 963739

31. Hurtubise V and Freeds K F 1993 Adv. Chem. Phys. 83 465

32. Durand P and Malrieu J P 1987 Adv. Chem. Phys. 67321

33. Evangelisti S, Daudey J P and Malrieu J P 1987 Phys. Rev. A: At. Mol. Opt. Phys. 354930

34. Jeziorski B and Monkhorst H J 1981 Phys. Rev. A: At. Mol. Opt. Phys. 241668

35. Paldus J, Pylypow L and Jeziorski B 1989 Many-body methods in quantum chemistry, Lecture notes in chemistry (ed.) U Kaldor, vol 52, p. 151

36. Balkova A, Kucharski S A, Meissner L and Bartlett R J 1991 J. Chem. Phys. 954311

37. Kutzelnigg W 1982 J. Chem. Phys. 772081

38. Mukherjee D, Moitre R K and Mukhopadhyay A 1975 Mol. Phys. 301861

39. Mukherjee D 1979 Pramana 12203

40. Mukherjee D and Pal S 1989 Adv. Quantum Chem. 20 292

41. Lindgren I and Mukherjee D 1987 Phys. Rep. 15193

42. Haque M and Kaldor U 1985 Chem. Phys. Lett. 117 347

43. Pal S, Rittby M, Bartlett R J, Sinha D and Mukherjee D 1988 J. Chem. Phys. 884357

44. Meissner L, Jankowski K and Wasilewski J 1996 J. Chem. Phys. 1046582

45. Chattopadhyay S, Mahapatra U S, Dutta B and Mukherjee D 2002 Chem. Phys. Lett. 357426

46. Chattopadhyay S, Mahapatra U S and Mukherjee D 1999 J. Chem. Phys. 1113820
47. (a) Ben-shloma S and Kaldor U 1988 J. Chem. Phys. 89 956; (b) Koch S and Mukherjee D 1988 Chem. Phys. Lett. 145321

48. Geertsen J, Rittby M and Bartlett R 1989 J. Chem. Phys. Lett. 16457

49. Sekino H and Bartlett R J 1984 Int. J. Quantum Chem. S18 255

50. Bartlett R J and Stanton J F 1994 Reviews in computational chemistry (eds) K B Lipkowitz and D B Boyd (New York: VCH) Vol. 5, p. 65

51. Pieniazek P A, Arnstein S A, Bradforth S E, Krylov A I and Sherrill C D 2007 J. Chem. Phys. 127164110

52. Nooijen M and Bartlett R J 1995 J. Chem. Phys. 102 3629

53. Kowalski K and Piecuch P 2001 Chem. Phys. Lett. 347 237

54. (a) Koch H and Jorgensen P 1990 J. Chem. Phys. 93 3333; (b) Koch H, Jensen H, Jorgensen P and Helgeker T 1990 J. Chem. Phys. 933345

55. (a) Mukherjee D and Mukherjee P K 1979 Chem. Phys. 39 325; (b) Ghosh S, Mukherjee D and Bhattacharyya S N 1981 Mol. Phys. 43 173; (c) Ghosh S, Mukherjee D and Bhattacharyya S N 1981 Mol. Phys. 43 173; (d) Harris F E 1977 Int. J. Quantum Chem. S11 403

56. (a) Nooijen A and Bartlett R J 1997 J. Chem. Phys. 106 6441; (b) Nooijen A and Bartlett R J 1997 J. Chem. Phys. 1066449

57. Krylov A I 2001 Chem. Phys. Lett. 338375

58. Pal S 1989 Phys. Rev. A: At. Mol. Opt. Phys. 3939

59. Pal S 1992 Int. J. Quantum Chem. 41443

60. (a) Ajitha D and Pal S 2001 J. Chem. Phys. 114 3380; (b) Ajitha D, Vaval N and Pal S 1999 J. Chem. Phys. 110 2316; (c) Ajitha D and Pal S 1999 Chem. Phys. Lett. 309 457; (d) Vaval N and Pal S 1999 Chem. Phys. Lett. 300 125

61. Ajitha D and Pal S 1997 Phys. Rev. A 562658

62. Shamasunder K R and Pal S 2001 J. Chem. Phys. 114 1981

63. Shamasunder K R and Pal S 2004 J. Chem. Phys. 120 6381

64. Manohar P U, Vaval N and Pal S 2006 J. Mol. Struct. Theochem. 76891

65. Manohar P U and Pal S 2007 Chem. Phys. Lett. 438 321

66. Bag A, Manohar P U, Vaval N and Pal S 2009 J. Chem. Phys. 131024102

67. Ravichandran L, Vaval N and Pal S 2009 Int. J. Quantum Chem. 1092191

68. Christiansen O, Jorgensen P and Hattig C 1998 Int. J. Quantum Chem. 681

69. Stanton J F 1993 J. Chem. Phys. 998840

70. (a) Staton J F and Gauss J 1994 J. Chem. Phys. 101 8938; (b) Stanton J F and Gauss J 1995 J. Chem. Phys. 10388931

71. Stanton J F and Bartlett R J 1993 J. Chem. Phys. 98 7029

72. (a) Nooijen M and Bartlett R J 1997 J. Chem. Phys. 107 6812; (b) Gwaltney S R, Bartlett R J and Nooijen M 1999 J. Chem. Phys. 11158

73. Levchenko S V, Wang T and Krylov A I 2005 J. Chem. Phys. 122224106

74. Kowalski K and Piecuch P 2002 J. Chem. Phys. 116 7411 
75. Pieniazek P A, Bradforth S E and Krylov A I $2008 \mathrm{~J}$. Chem. Phys. 129074104

76. Kallay M and Gauss J 2004 J. Chem. Phys. 1219257

77. Kamiya M and Hirata S 2006 J. Chem. Phys. 125 074111

78. Musial M, Kucharski S A and Bartlett R J 2003 J. Chem. Phys. 1181128

79. Kowalski K and Piecuch P 2002 J. Chem. Phys. 113 8490

80. Evangelista F A, Prochnow E, Gauss J and Schaefer H F III 2007 J. Chem. Phys. 132074107

81. Stanton J F and Gauss J 1996 Theor. Chim. Acta 93303

82. Manohar P U, Stanton J F and Krylov A I 2009 J. Chem. Phys. 131114112

83. Manohar P U and Krylov A I 2008 J. Chem. Phys. 129 194105

84. Kowalski K and Piecuch P 2001 J. Phys. Chem. 115 643

85. (a) Scuseria G E 1991 J. Chem. Phys. 94 442; (b) Gauss J and Stanton J F 1997 Chem. Phys. Lett. 27670

86. Gauss J and Stanton J F 2002 J. Chem. Phys. 1161773

87. Ravichandran L, Vaval N and Pal S 2011 J. Chem. Theory Comput. 7876
88. Baluja K L and Tossell J A 2003 J. Phys. B 3619

89. (a) ACES II is a program product of the Quantum Theory Project, University of Florida. Authors: Stanton J F, Guass J, Perera S A, Watts J D, Yau A D, Oliphant N, Szalay P G, Lauderdale W J, Gwaltney S R, Beck S, Balkova A, Nooijen M, Sekino H, Huber C, Baeck K K, Bernholdt D E, Rozyczko P, Pittner J, Cencek W, Taylor D and Bartlett R J; (b) Integral package included is GAMESS, Authors: Schmidt M W, Baldridge K K, Boatz J A, Elbert S T, Gordon M S, Jensen J J, Koseki S, Matsunagu N, Nguyen K A, Su S, Windus T L, Dupuis $\mathrm{M}$ and Montgomery $\mathrm{J}$ A

90. Amano T, Saito S, Hirota E and Morino Y $1969 \mathrm{~J} . \mathrm{Mol}$. Spectrosc. 30275

91. (a) Chong D P 1994 Chem. Phys. Lett. 220 102; (b) Petersson L G M, Langhoff S R and Chong D P $1986 \mathrm{~J}$. Chem. Phys. 852836

92. Amano T and Hirato E 1973 J. Mol. Spectrosc. 45417

93. Staemmler V 1982 Theor. Chim. Acta 6269

94. Byfleet C R, Carrington A and Russel D K $1971 \mathrm{Mol}$. Phys. 20271

95. Yaron D, Peterson K and Klemperer W 1988 J. Chem. Phys. 884702 